\title{
Correction to: Neuroimmune Response in Ischemic Preconditioning
}

\author{
Ashley McDonough $^{1}$ • Jonathan R. Weinstein ${ }^{1}$
}

Published online: 6 November 2017

(C) The American Society for Experimental NeuroTherapeutics, Inc. 2017

\begin{abstract}
Ischemic preconditioning (IPC) is a robust neuroprotective phenomenon in which a brief period of cerebral ischemia confers transient tolerance to subsequent ischemic challenge. Research on IPC has implicated cellular, molecular, and systemic elements of the immune response in this phenomenon. Potent molecular mediators of IPC include innate immune signaling pathways such as Tolllike receptors and type 1 interferons. Brain ischemia results in release of pro- and anti-inflammatory cytokines and chemokines that orchestrate the neuroinflammatory response, resolution of inflammation, and transition to neurological recovery and regeneration. Cellular mediators of IPC include microglia, the resident central nervous system immune cells, astrocytes, and neurons. All of these cell types engage in cross-talk with each other using a multitude of signaling pathways that modulate activation/ suppression of each of the other cell types in response to ischemia. As the postischemic neuroimmune response evolves over time there is a shift in function toward provision of trophic support and neuroprotection. Peripheral immune cells infiltrate the central nervous system en masse after stroke and are largely detrimental, with a few subtypes having beneficial, protective effects, though the role
\end{abstract}

Due to a technical error in the production process, the earlier version of this article contained numerous errors in the reference numbering. We are reprinting the entire article in the correction for readability

The online version of the original article can be found at https://doi.org/ 10.1007/s13311-016-0465-z

Jonathan R. Weinstein

jweinste@u.washington.edu

1 Department of Neurology, University of Washington, Seattle, WA, USA of these immune cells in IPC is largely unknown. The role of neural progenitor cells in IPC-mediated neuroprotection is another active area of investigation as is the role of microglial proliferation in this setting. A mechanistic understanding of these molecular and cellular mediators of IPC may not only facilitate more effective direct application of IPC to specific clinical scenarios, but also, more broadly, reveal novel targets for therapeutic intervention in stroke.

Keywords Ischemic preconditioning - Microglia . Interferon $\cdot$ Toll-like receptor $\cdot$ Stroke $\cdot$ Progenitors

\section{Correction to: Neurotherapeutics (2016) 13:748-761 https://doi.org/10.1007/s13311-016-0465-Z}

\section{Preconditioning}

Ischemic preconditioning (IPC) is an experimental phenomenon in which a brief period of ischemia confers robust neuroprotection against subsequent ischemic events [1-3]. It is important to note that preconditioning does not reduce the incidence of stroke, but rather ameliorates the pathophysiologic response to cerebral ischemia and results in a smaller infarct volume and improved poststroke recovery [4-6]. Clinical studies suggest that patients with stroke who suffered a recent prior transient ischemic attack have better outcomes than those who did not, implying that a human correlate to experimental IPC exists [7, 8]. Elucidating the mechanisms of IPC is considered a critical challenge in stroke research $[9,10]$. Recent literature has implicated innate immune pathways, including Toll-like receptors (TLRs) $[11,12]$ and type 1 interferon (IFN) signaling $[5,11,13]$ in IPC-mediated protection. 
IPC induces a reprogramming of the transcriptional response to stroke, which results in a neuroprotective phenotype, rather than the destructive inflammatory phenotype observed after stroke alone [4, 6, 14]. After stroke alone, genes that coordinate immune and stress responses are upregulated in the brain, but after preconditioning and preconditioning followed by stroke, genes in metabolism and transport/ synaptic transmission are downregulated [14]. The response to preconditioning is one of reduced cellular activity, and is highly similar to changes in gene expression seen in hibernating animals $[14,15]$. Furthermore, the gene expression observed after preconditioning plus stroke is substantially different from that observed after stroke alone [6]. Within this review we will discuss this phenomenon of preconditioning, with a particular focus on preconditioning against stroke and cellular mediators of this phenomenon.

Preconditioning has three phases: a priming phase that establishes protection, a refractive phase during which the system is resistant to ischemic injury, and a neuroprotective phase that is characterized by an altered response to stroke that reduces injury [9]. The refractive phase is of short duration, lasting 1 to 7 days, depending on the preconditioning treatment $[3,4,6,9]$. The three phases, and their molecular effectors and characteristics, have been reviewed elsewhere in detail [9], and are summarized in Table 1. Within this review, we will focus on the most important molecular mediators of preconditioning, as well as cellular mediators of preconditioning effects.

\section{Cross-Tolerance}

IPC primes the brain against subsequent ischemic injury. This concept of preconditioning has correlates in other forms of injury, such as a brief epileptic seizure preconditioning the brain against a subsequent longer seizure that may be injurious, or even against other ischemic events. Interestingly, one form of preconditioning may protect the brain against other types of injuries $[1,2]$. One example of this is lipopolysaccharide (LPS)-induced cross-tolerance, whereby challenging the immune system with the bacterial cell membrane component LPS (or endotoxin) preconditions the brain against stroke $[5,6]$. This appears to be owing, in large part, to the importance of TLR4 in response to LPS or ischemia [4-6, 12, 29]. However, while there is overlap in the genetic response to LPS or IPC, there is a substantial subset of genes unique to each preconditioning stimulus [6]. The LPS preconditioning response largely focuses on modulating inflammatory processes [6], while IPC results in both metabolic changes to protect against injury from low oxygen [26], and also modulates the neuroimmune response through mechanisms such as TLRs and IFN signaling pathways $[5,9,29]$. The shared modulation of neuroimmune components appears to be responsible for the overlap in neuroprotection offered against ischemia by multiple preconditioning stimuli.

Table 1 Overview of selected molecular mediators expressed at different phases of the preconditioning response

\begin{tabular}{|c|c|c|c|}
\hline Phase & Molecular mediators & Functions & Cells types \\
\hline \multirow[t]{4}{*}{ Priming } & TLRs & $\begin{array}{l}\text { Induce inflammatory serum cytokines and } \\
\text { transcription factors, i.e., TNF- } \alpha \text {, IL-1, IL-6, NFkB }\end{array}$ & $\begin{array}{l}\text { Circulating leukocytes [16], endothelial cells [16], } \\
\text { microglia }[17,18]\end{array}$ \\
\hline & TNF- $\alpha$ & Induces low-level inflammatory response in the CNS & $\begin{array}{l}\text { Astrocytes }[19,20], \text { microglia [20], peripheral } \\
\text { immune cells [19-21] }\end{array}$ \\
\hline & HIF-1 & Regulates metabolic genes to protect against hypoxia & $\begin{array}{l}\text { Multiple CNS cells }[17,22] \text {, peripheral } \\
\text { immune cells [23] }\end{array}$ \\
\hline & Type 1 IFNs & Regulate neuroimmune function & Microglia [11], peripheral macrophages [24, 25] \\
\hline \multirow[t]{3}{*}{ Refractive } & microRNAs & Modulate gene expression post-transcriptionally & Multiple CNS cells $[18,26]$ \\
\hline & $\begin{array}{l}\text { Polycomb group } \\
\text { proteins histone } \\
\text { modulators }\end{array}$ & Induce lasting alterations of gene-transcription programs & Multiple CNS cells $[18,26]$ \\
\hline & TNF- $\alpha$ & Neuroprotectant and/or proapoptotic & $\begin{array}{l}\text { Astrocytes [19-21], microglia [19-21], } \\
\text { peripheral immune cells [19-21] }\end{array}$ \\
\hline \multirow[t]{3}{*}{ Neuroprotective } & IRFs & $\begin{array}{l}\text { Downstream mediators of the reprogrammed } \\
\text { genomic response to ischemia }\end{array}$ & Microglia [11], macrophages [11] \\
\hline & TLRs & Reprograms cellular genomic response to ischemia & $\begin{array}{l}\text { Circulating leukocytes [16], endothelial cells [16], } \\
\text { microglia }[17,18]\end{array}$ \\
\hline & TGF- $\beta$ & $\begin{array}{l}\text { Promotes repair of neurovascular unit, regulates } \\
\text { immune system function }\end{array}$ & Astrocytes [20, 27], microglia [27], T cells [28] \\
\hline
\end{tabular}

TLR = Toll-like receptor; TNF = tumor necrosis factor; NFkB = nuclear factor $\mathrm{kB} ; \mathrm{CNS}=$ central nervous system; HIF = hypoxia-inducible factor; IFN = interferon; IRF $=$ IFN regulatory factor; TGF $=$ transforming growth factor 


\section{Remote Preconditioning}

There are multiple strategies for inducing preconditioning effects in the central nervous system (CNS) as a protective mechanism for patients at risk of stroke. Inducing a controlled short ischemic event in the clinic poses a number of risks, so many studies have been geared at less invasive methods of inducing preconditioning. These include the administration of therapeutics, such as cytokines or TLR agonists which play a role in preconditioning, or the concept of remote IPC (RIPC), which uses remote limb ischemia to induce neuroprotection equivalent to direct preconditioning of the brain using models of focal ischemia $[4,30,31]$. The relative ease of administration, low cost, and potential wide generalizability of RIPC make the concept a particularly attractive clinical strategy for select populations of patients that are at high risk for cerebral ischemic events in the immediate (hours to days) future. This would include patients that are about to undergo major cardiac $[32,33]$ or carotid artery [34] surgery, as well as possibly patients with subarachnoid hemorrhage that are at high risk of developing cerebral vasospasm and accompanying delayed cerebral ischemia $[35,36]$. The pioneering papers in the RIPC field suggest that neuroprotection is induced rapidly $[30,31$, 37]; however, the duration of protection has not been determined $[37,38]$. Molecular determinants behind the phenomenon of RIPC have not been fully elucidated, but there is some evidence of overlap with molecular mediators of ischemic and LPS preconditioning, including hypoxia-inducible factor (HIF)- $1 \alpha$ [39], and inflammatory factors [40-43]. Remote limb ischemia may induce global expression of these molecular mediators of preconditioning that confers protection to multiple organ systems, including the brain. Some studies of remote limb ischemia have demonstrated reduced inflammation in multiple organs after preconditioning [42, 43], which suggests that ischemic protection via modulation of immune responses may extend to the entire body and not just be localized to a single organ or region of the body.

Recent clinical trials on the efficacy of RIPC found no significant difference in composite endpoints in patients treated with RIPC and those which received sham RIPC treatment prior to cardiac surgery $[32,33]$. These trials were large, randomized, controlled, double-blinded, highly powered studies with carefully selected patient cohorts from multiple treatment centers. However, the chosen endpoint parameters evaluated in each were patient mortality, myocardial infarction, stroke, and acute renal failure. These negative results are not particularly surprising because, based on our current understanding of preconditioning, we would not anticipate that IPC would have an effect on the outcome parameters measured. There is no evidence that IPC prevents stroke occurrence or reduces incidence of other thromboembolic events. One could, however, reasonably anticipate protective effects of RIPC on neurological outcomes and infarct volume [4-6]. These more difficult to assess outcome parameters have not yet been reported in the recent large trials. Thus, although these studies were rigorous in their controls and design, the chosen endpoint parameters did not reflect the biology and neurological effects of IPC, and the clinical efficacy of RIPC is undetermined with regard to these parameters.

\section{Molecular Mediators of Preconditioning}

Preconditioning involves neuroimmune signaling. Several lines of evidence implicate innate immune signaling in IPCmediated neuroprotection. Pre-exposure to LPS, a primary ligand for TLR4 and a potent immune cell activator, induces a form of neuroprotection called cross-tolerance that is similar to IPC [44]. IPC-mediated neuroprotection is itself attenuated in TLR $4^{-/-}$mice $[11,12]$, and agonists of multiple other TLRs also induce cross-tolerance [45-47]. Genomic studies on preconditioned cortical tissue have identified robust expression of IFN-stimulated genes (ISGs) [5, 13] - a well-defined innate immune response activated by type 1 IFNs such as IFN- $\alpha$ and IFN- $\beta$. Both IPC and TLR agonist-induced cross-tolerance are attenuated in mice with deficiencies in IFN signaling [13]. We provide evidence here that IPC results in marked changes in the innate immune cell population in cortex (Fig. 1), even in the absence of infarction. We have also recently found and confirmed robust ISG expression in ex vivo flow cytometrically sorted microglia from preconditioned cortex (A. McDonough and J.R Weinstein, unpublished observations). Furthermore, we have demonstrated in a novel white matter model of IPC, that neuroprotection is abolished by genetic knockdown of type 1 IFN receptor (IFNAR1) specifically in microglia [11]. These findings have provided strong evidence to support a key role for innate immune signaling and microglia in preconditioning-mediated neuroprotection. The molecular pathways in IPC have been recently reviewed extensively [10]. Here we will focus selectively only on some of the most important and well-defined molecular mediators of preconditioning.

Type 1 IFNs are key cytokines in the innate immune system, including 13 IFN- $\alpha$ subtypes, as well as IFN- $\beta$, and signal through the IFNAR1 receptor complex $[49,50]$. Although type 1 IFNs are classically upregulated in response to viral infection, recent studies have implicated them as key regulators of the neuroimmune response triggered by noninfectious causes of CNS injury [51]. Recently Inacio et al. [52] found that endogenous IFN- $\beta$ signaling exerts antiinflammatory actions in experimentally induced focal cerebral ischemia [52]. Type 1 IFNs have also been implicated in LPS preconditioning [5], and IFN- $\beta$ levels increase in the brain after LPS preconditioning followed by stroke but not by stroke alone [5]. In a study comparing wild-type and IFN- $\beta^{-/-}$mice, there were no significant differences in infarct volume after middle cerebral artery occlusion (MCAO) 

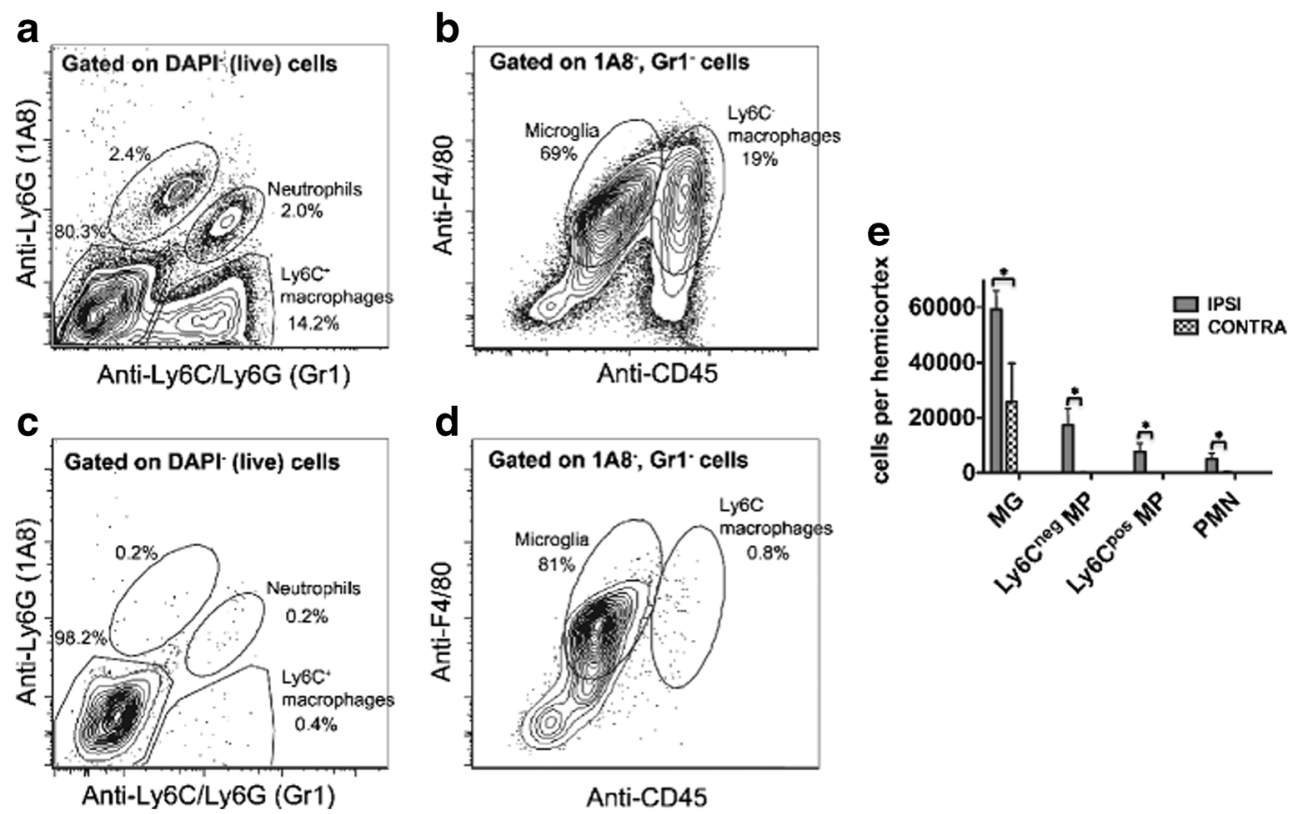

Fig. 1 Peripheral immune cells infiltrate the ipsilateral cortex after ischemic preconditioning. Ex vivo flow cytometry was performed on the (a, b) ipsilateral (IPSI) and (c, d) contralateral (CONTRA) hemicortices $72 \mathrm{~h}$ after a 15 -min middle cerebral artery occlusion (ischemic preconditiong pulse). The number of cells is quantified in (E), and demonstrates an increase in the number of microglia (MG), as well as an

influx of Ly6 $\mathrm{C}^{\text {neg }}$ and $\mathrm{Ly} 6 \mathrm{C}^{\text {pos }}$ macrophages (MP), as well as polymorphonuclear neutrophils (PMN) into the ipsilateral, but not the contralateral, hemicortex after a preconditioning stimulus $[n=17$ mice (4 separate ex vivo flow cytometry preparations) $\left.{ }^{*} p<0.01\right]$. Experiments were performed as previously described [48]

(stroke) [5], suggesting that IFN- $\beta$ is not an outcomedetermining factor in the acute stroke response. However, when IFN- $\beta$ was administered prior to MCAO, there was a $35 \%$ reduction in infarct volume [5], suggesting that type 1 IFNs can protect the brain against subsequent ischemia. IFN regulatory transcription factors (IRF3 and IRF7) downstream from IFNAR1 signaling are critical for IPC-mediated protection in gray matter infarct volume models of IPC [13]. As noted above, IPC-mediated protection in white matter was entirely dependent on type 1 IFN signaling [11]. However, the full extent and mechanisms of IFN signaling in IPCmediated protection remain to be explored. The cellular source of IFN- $\beta$ in the postischemic brain is unknown, but there are several possible sources such as peripheral macrophages [53, 54] and astrocytes [55-58]. Published studies also suggest neurons [59] are capable of releasing IFN- $\beta$ under specific conditions.

TLRs are a family of pattern recognition receptors involved in the identification of, and response to, foreign pathogens [16]. To date, 13 TLRs have been identified, and each recognizes different pathogen-associated molecular patterns $[16,60]$. TLRs are expressed on antigen presenting cells and are critical in the innate immune response [16]. The TLR family includes receptors for bacterial cell wall/membrane components such as lipotechoic acid (TLR2), peptidoglycan (TLR2), and LPS (TLR4) [16]. Activation of TLRs by endogenous ligands, also known as danger-associated molecular patterns (DAMPs), released from ischemia-injured cerebral vasculature and parenchyma is a possible mechanism for initiation of inflammatory responses in stroke [2, 61]. A number of putative DAMPs, including heat shock proteins (HSPs) [62, 63], especially HSP70 [64] and HSP60 [62] — both will activate TLRs - have been identified in the brain. Systemic administration of ligands for multiple TLRs (reviewed by Stevens et al. [9]) reduces ischemic injury in rodent models of adult and neonatal ischemia, and pharmacological preconditioning with a TLR agonist demonstrably provides protection against stroke in primates [65]. Activation of TLRs is also known to induce expression of type 1 IFNs in a number of cell types, including monocytes and dendritic cells [24, 25]. However, while TLRs appear to play a protective role in preconditioning, TLRs are also implicated in damaging pathways within the context of acute stroke via the activation of nuclear factor $\mathrm{kB}[66$, 67]. Engagement of TLR signaling after ischemia may largely depend on the ischemic context, that is, a brief period of ischemia (IPC) versus a longer ischemic event (stroke), with shifting kinetics as the response evolves from injurious to protective over time $[9,68]$.

$H I F-1$ is an upstream regulator for a series of hypoxiaresponsive genes, including glucose transporter (GLUT) 1 and vascular endothelial growth factor (VEGF), and is upregulated by hypoxic preconditioning $[4,22,69]$. In astrocytes, HIF-1 also induces the expression of $\mathrm{P} 4502 \mathrm{C} 11$, an arachidonic acid epoxygenase, which is protective against ischemia-reperfusion injury in multiple organ systems [22], and 
contributes to IPC-mediated protection [22]. HIF-1 controls the glycolytic response of immune cells and profoundly alters inflammatory responses under conditions of hypoxia [23]. Microglia will express HIF-1 in response to hypoxic culture conditions [17]. There is some cross-talk between the TLR and HIF-1 signaling pathways as pathogen-associated molecular patterns like LPS can induce HIF-1 expression in microglia and other cell types [17].

Tumor necrosis factor (TNF)- $\alpha$ may be released by a multitude of cell types after ischemic injury [19], and has roles as both a neuroprotectant and proapoptotic agent. Astrocytes release TNF- $\alpha$ after ischemic and excitotoxic brain injury [21]. LPS injection also induces secretion of TNF- $\alpha$ by microglia within 2 to $4 \mathrm{~h}$ [20]. Furthermore, when activated by oxygenglucose-deprived (OGD) neurons, microglia release TNF- $\alpha$ [70]. Responding peripheral immune cells also release TNF- $\alpha$ in response to ischemia $[19,71]$. TNF- $\alpha$ is required for cross-tolerance induced by multiple TLR agonists [72, 73]. Multiple TLRs induce TNF- $\alpha$ in the brain, which appears to correlate with protective effects of cross-tolerance [9].

Transforming growth factor (TGF)- $\beta$ is a neuroprotectant released by multiple cell types. Astrocytes release TGF- $\beta$ in response to ischemia, which protects both astrocytes and neurons [21]. TGF- $\beta$ is also released by astrocytes within $8 \mathrm{~h}$ of LPS injection [20]. TGF- $\beta$ may also be released by macrophages to promote repair of the neurovascular system after stroke [74], and may also be released by $\mathrm{T}$ cells in the later stages of recovery from ischemia [19]. There is also evidence that microglia secrete TGF- $\beta$ at later stages of recovery following stroke when they are functioning in a neuroprotective mode [71].

\section{Cellular Mediators of IPC}

The cellular response to stroke, particularly the response of immune cells, has been studied extensively and has been recently reviewed in detail $[19,75]$. Post-ischemic inflammation is characterized by an orderly sequence of events involving the brain, its vessels, the circulating blood, and lymphoid organs [19]. The responses begin in the intravascular compartment and include release of inflammatory mediators such as cytokines, chemokines, proteases, and small vasoactive compounds (including prostanoids, leukotrienes, and adenosine triphosphate) that induce multiple changes in endothelial cell and leukocyte function. These changes result in blood-brain barrier (BBB) breakdown and leukocyte infiltration into the brain parenchyma. There is subsequent initiation of innate immune responses in the ischemic penumbra by microglia, macrophages, and astrocytes through activation of pattern recognition receptors, including the TLRs, by DAMPs. T-cellbased and adaptive immune responses are then initiated and can be broken down both temporally (acute $v s$ delayed phases) and functionally (detrimental vs protective) [19]. Numerous active cellular processes and complex cellular interactions contribute to the resolution of postischemic inflammation. These processes include clearing of dead cells by microglia and infiltrating macrophages, release of anti-inflammatory cytokines such as TGF- $\beta$ and interleukin (IL)-10 by microglia and macrophages, as well as elaboration of growth factors such as insulin-like growth factor 1 and VEGF by astrocytes and neurons. Insulin-like growth factor 1 and VEGF contribute to postischemic neuronal sprouting and angiogenesis, respectively. Stroke also induces profound changes in the systemic immune response [75]. Within a few hours of the onset of cerebral ischemia, brain-immune system interactions can result in downregulation of systemic immunity, a phenomenon known as stroke-induced immunodepression [76]. Ischemia triggered systemic immune responses and strokeinduced cellular changes in the neurovascular unit and brain parenchyma define key elements of post-stroke pathophysiology and recovery. Many of these processes also play a central role in preconditioning-mediated neuroprotection. The kinetics of post-stroke immune reactions are critical in postischemic physiology and the concept of a biphasic or multiphasic response to brain ischemia is now favored [53, $68,75,77]$. The preconditioning phenomenon has this temporal component built into its structure such that the primary preconditioning stimulus (whether it is brain ischemia, remote ischemia, administration of a TLR agonist, or something else) induces a response that has already evolved considerably toward the "resolution of inflammation" or "regeneration and repair" phase. Below we review some of the cell type-specific responses that are central in preconditioning with emphasis on cellular immune responses, and a summary of these interactions is illustrated in Fig. 2.

\section{Microglia}

Microglia are CNS-resident immune cells derived from yolk sac macrophages that enter the CNS during early development and maintain themselves as a distinct population from circulating macrophages/monocytes [78-80], despite a high overlap in shared expression of many immunohistochemical markers [17, 81]. Microglia contribute to the maintenance of brain homeostasis, suggesting a critical role for microglia in the normal physiology of the CNS [56], and pathway analysis of baseline microglial gene expression (from naïve wild-type mouse brain) revealed that the functions most associated with microglia were related to nervous system development [82].

Microglia play a significant role in the neuroinflammatory response to ischemia $[17,18]$. The expression of multiple TLRs (TLRs 1-9) by microglia enables them to identify multiple pathogens and upregulate a unique profile of innate and effector immune cytokines and chemokines in response to a wide range of stimuli [83]. Most abundantly expressed by 


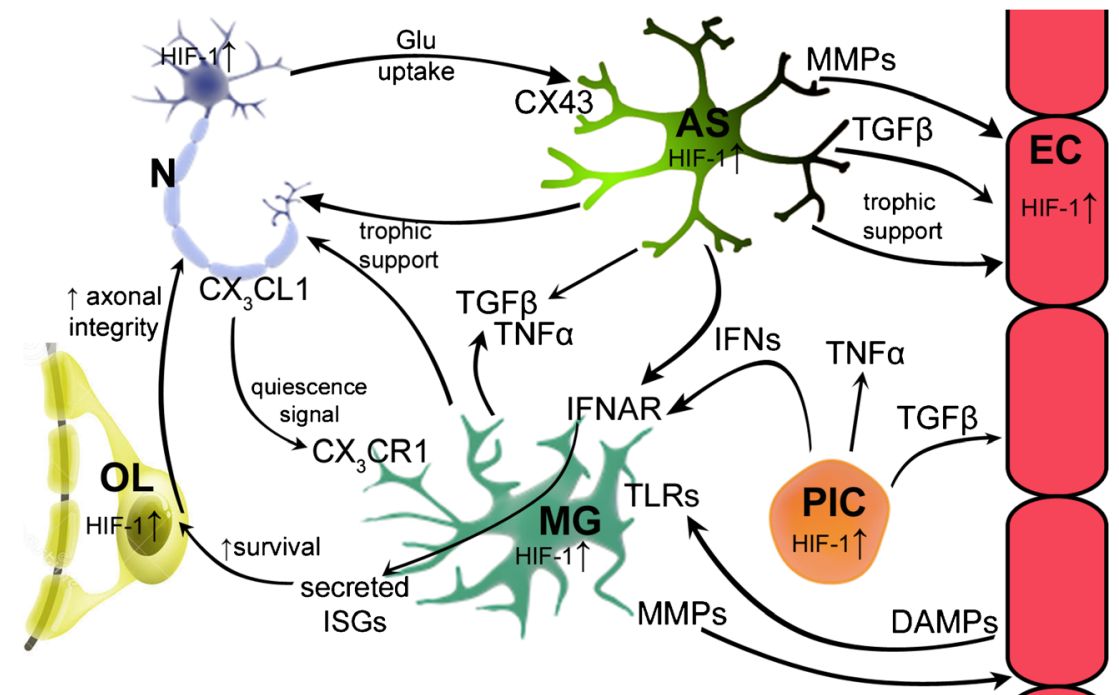

Fig. 2 Summary of key neuroimmune preconditioning pathways and interactions between cells of the central nervous system after ischemic preconditioning. Astrocytes (AS) provide trophic support to neurons (N) through multiple mechanisms, including uptake of glutamate (Glu) and secretion of $\operatorname{TGF} \beta$, which is also reparative to endothelial cells (EC). Astrocytes also provide trophic support to endothelial cells. Both microglia (MG) and astrocytes secrete TGF $\beta$ and TNF $\alpha$ in response to transient ischemia which may have protective effects during the refractive and neuroprotective phase of preconditioning. Neurons also signal via fractalkine $\left(\mathrm{CX}_{3} \mathrm{CL} 1\right)$ to microglia, which express cognate receptor $\mathrm{CX}_{3} \mathrm{CR} 1$. Both astrocytes and peripheral immune cells (PIC) are potential

microglia is TLR4, and both endogenous and exogenous TLR4 agonists potently activate classical proinflammatory responses in microglia $[18,83]$. Although microglial activation has typically been considered a proinflammatory process, recent publications suggest that microglia could play a protective role in stroke $[17,18,84,85]$ through multiple mechanisms such as metabolic and physiological support of neurons [86], production of trophic factors [85], phagocytosis of damaged cells and debris and repair of lesioned tissue by releasing matrix metalloproteinases (MMPs) [87]. While some of these responses, particularly the release of MMPs, can disrupt the $\mathrm{BBB}$ and be deleterious, microglia are also capable of regulating these processes and downmodulating the inflammatory response to a stimulus. Microglia are the first responders to ischemic injury, activating before peripheral monocytes/ macrophages infiltrate the CNS [88]. Similarly, in a recent study on microglial activation in response to LPS challenge, Norden et al. [20] found that microglial activation preceded astrocyte activation. The microglial response to LPS was rapid, with a robust induction of proinflammatory cytokine and chemokine mRNAs detected 2 to $4 \mathrm{~h}$ after LPS injection, which correlated with the onset of sickness behavior [20]. Ischemia and LPS induce markedly disparate genomic and phenotypic responses in microglia [17]; however, as discussed above in the section on cross-tolerance, LPS challenge can provide preconditioning against stroke. sources of type 1 interferons (IFNs) that signal to microglia via type I IFN receptor (IFNAR), triggering release of IFN-stimulated genes (ISGs). ISG protein products may enhance oligodendrocyte (OL) viability in the setting of prolonged ischemia and, in turn, increase axonal integrity in white matter. Endothelial cells are one of many that release danger-associated molecular patterns (DAMPs), which are ligands for numerous Toll-like receptors (TLRs). Peripheral immune cells are capable of secreting many different cytokines, which have effects on multiple cell types, for example TGF $\beta$ and type 1 IFNs. HIF-1 = hypoxia-inducible factor 1 ; MMP = matrix metalloproteinase

Microglia are also key cellular targets for IFN signaling in the CNS $[51,89,90]$ as they express IFNAR1 [11] and respond to type 1 IFNs (or type 1 IFN inducers such as the TLR3 agonist poly-IC) with robust expression of ISGs [51, 89, 91, 92]. Current data point to important type 1 IFNmediated alterations of microglial function in neuroinflammation [93]. IFN- $\beta$ reduces the antigen-presenting capacity of microglia, which inhibits the effector function of T cells [89, 94], and also induces changes in cytokine production that affects recruitment of peripheral immune cells into the CNS $[89,95]$. Other type 1 IFNs modulate the expression of nitric oxide and glutamate, which decreases the incidence of microglia-mediated neuronal death [96, 97]. IRFs, downstream of IFN signaling, play a crucial role in the polarization of microglia and macrophages [98-100]. Furthermore, IFN- $\beta$ enhances the ability of microglia to phagocytose apoptotic $\mathrm{T}$ cells, thus resulting in a modulation of the peripheral immune response [101]. Finally, as noted above, IPC-mediated protection in white matter was eliminated by cell-targeted knockdown of specific gene expression (ifnarl) in microglia [11].

\section{Astrocytes}

IPC demonstrably protects astrocytes against longer periods of ischemia $[69,102]$. Hypoxic preconditioning of astrocytes in culture leads to greater survivability and increases in 
glucose transport over nonpreconditioned astrocytes, primarily through increased levels of GLUT1 and GLUT3 after prolonged hypoxia [69]. Other studies have demonstrated that after IPC, astrocytes upregulate 14-3-3 $\gamma$ [102, 103], a multifunctional scaffolding protein, and alleviate energy depletion through different pathways [102]. Ischemia induces a cellular redistribution of astrocytic gap junction proteins, including Cx34 [104, 105]. Cx34-expressing astrocytes have been found in the peri-infarct region of the poststroke brain [104], where they may secrete growth factors that promote neuronal survival and stimulate angiogenesis in infarcted tissue [105-107].

Much like microglia, astrocytes are capable of responding to inflammation and exhibit activated states that correspond to a change in cellular morphology and gene expression [21]. Also similar to microglia, astrocytes are capable of performing destructively by releasing high levels of nitric oxide, glutamate, TNF- $\alpha$, and/or other neurotoxic cytokines [21], or may confer neuroprotection through trophic support [21, 105]. Reactive astrocytes express glial fibrillary acidic protein (GFAP) and vimentin (Vim) [103]. In a study of GFAP knockout, Vim knockout, and double knockout mice examining responses to permanent $\mathrm{MCAO}$, the double knockout mice $\left(\mathrm{GFAP}^{-/} ; \mathrm{Vim}^{-1}\right.$ ${ }^{-}$) had a 2 to 3.5-fold increase in infarct volume compared with single knockouts and wild-type controls [103], suggesting that reactive astrocytes may have a protective role in brain ischemia. Knockout of both GFAP and Vim also reduced glutamate uptake by $44 \%$ [103]. A hallmark of these impaired astrocytes is their inability to form intermediate filaments (IFs) in response to outside stimuli [103], such as ischemia. However, how IFs confer protection in ischemia is unclear, as there are several pathways impaired in IF-deficient astrocytes related to normal astrocytic functions, such as glutamate uptake, cell-cell signaling, and neurotransmission [103].

Recent publications suggest a close relationship between microglia and astrocytes, as well as tightly coupled sequential activation of these two cell types. In a time course study of responses to peripheral LPS challenge, Norden et al. [20] found that microglia are activated first, followed by astrocytes. Furthermore, microglial IL-10 stimulates astrocytic TGF- $\beta$ in response to LPS challenge [27]. The large overlap between molecular pathways activated in response to LPS and ischemia is, in part, responsible for the phenomenon of crosstolerance (discussed previously), suggesting that IPC may modulate microglia/astrocyte interplay, with both cells playing important roles in conferring IPC-mediated neuroprotection. Astrocytes express TLR4 [108], a critical component in IPC-mediated neuroprotection [11, 12]. Activation of astrocytic TLR4 results in activation of nuclear factor $\mathrm{kB}$ and signal transducer and activator of transcription 3 in astrocytes [109], and downstream of this pathway is growth-associated protein (GAP)43, which is a protein kinase C-activated phosphoprotein implicated in axonal plasticity and regeneration
[109]. GAP43 is important for the glutamate uptake activity in reactive astrocytes following LPS challenge [109] or ischemic conditions [21, 69, 107]. Additionally, TLR3 expression in astrocytes appears to be critical to the astrocytic response to IPC [110]. Thus, many of the molecular pathways implicated in LPS studies of astrocytic activation and response may overlap with findings in IPC-mediated astrocytic activation.

In a different model of neuroinflammation, within the context of Alzheimer's disease, there is evidence of astrocytemicroglia cross-talk through the complement system [111]. Astrocytes are capable of synthesizing essentially all complement activation proteins, regulatory molecules, and receptors [21, 111]. Microglia express multiple complement receptors, including CR3, and this signaling pathway greatly influences phagocytic function of microglia [112, 113]. There is also evidence that infiltrating immune cells may influence astrocyte activation and behavior [58], suggesting astrocytes may be an important mediator between resident and peripheral immune cells and neurons. Astrocytes also produce many regulatory factors that may provide negative feedback to microglia, helping to modulate microglial activation states. In one example, TGF- $\beta$ produced by astrocytes, in response to IL10 secretion by microglia, provides negative feedback on microglial activation [27]. In another study, knockdown of astrocytic GAP43 led to increased microglial activation in the context of LPS challenge [109], suggesting that GAP43 expression in astrocytes attenuates the LPS-based immune response in microglia.

In addition to microglial-astrocyte cross-talk, neurons and astrocytes also have a tight relationship. Astrocytes play a critical role in the maintenance of the extracellular environment and transfer of energy substrates to neurons [21, 114], to such an extent that neuronal survival is correlated with astrocyte survival [106], and neuron-astrocyte interactions are critical for the normal function of the CNS [114]. One potential mechanism of astrocyte-mediated neuroprotection after IPC may involve limiting neuronal excitotoxicity by clearing glutamate [21], and in stroke astrocytes may protect neurons from oxidative stress [103]. Astrocytes are capable of engaging in spatial buffering, which allows them to transport and metabolize amino acids and glucose, as well as upregulate antioxidants and free radical scavengers in the ischemic region [103, 107, 114-116]. Prion protein protects neurons against oxidative stress, hypoxia, ischemia, and hypoglycemia, and may be supplied to neurons by astrocytes under ischemic or hypoxic conditions [117].

Astrocytes can also influence preconditioning mediated neuroprotection through their multiple and potent effects on the microvasculature of the CNS. Astrocytic end feet wrap around blood vessels in the CNS where they are well poised to exert influences on the blood-brain barrier (BBB) after ischemia. Astrocytes are capable of secreting MMPs that disrupt the $\mathrm{BBB}$, or releasing trophic factors that protect the 
BBB. During cerebral ischemia, pericapillary astrocyte end feet are the first cellular elements to swell [118], and MMPs and IL- $1 \beta$ released by astrocytes contribute to BBB disruption and vasogenic edema $[119,120]$. Astrocytes may also encourage angiogenesis [106] and regulate vascular tone [121], both processes crucial in restoring blood flow to infarcted tissues. Several studies have demonstrated that inhibiting the reactivity and/or proliferation of astrocytes delays neurovascular remodeling after cerebral ischemia in rodents, and contributes to a worse functional outcome [103, 122, 123]. A recent study used repetitive pure oxygen exposure preconditioning to induce neuroprotection against stroke (MCAO) in a rodent model and found that the preconditioning stimulus activated astrocytes and increased BBB permeability [124]. Similar mechanisms of astrocyte-BBB interactions have not yet been characterized in the context of IPC, but many of the same mechanisms involving astrocytes may apply.

\section{Neurons}

Neurons that were exposed to conditioning via intermittent hypoxia prior to an extended period of hypoxia had upregulated glucose transport activity and increased viability compared with neuronal cultures that had been exposed to a lengthy period of hypoxia [69]. Inhibiting glucose transport with cytochalasin B abolished the hypoxic tolerance effect of preconditioning [69], suggesting this is an important consequence of preconditioning. Adenosine 5'-monophosphate-activated protein kinase is another major regulator of cellular energy dynamics, and is highly expressed in neurons [64]. As discussed previously in this review, preconditioned astrocytes exhibit increased glucose transport, and may protect neurons through spatial buffering, suggesting that multiple CNS cells independently, and in tandem with each other, undergo shifts in metabolic pathways and cell-cell signaling that enable these cells to survive a subsequent ischemic challenge (stroke) that occurs shortly after the preconditioning.

Neurons in hypoxic culture conditions have a distinct pattern of gene expression including genes coding for endoplasmic reticulum proteins, ubiquitination pathway components, proapoptotic factors, and other hypoxia-induced genes, including Bcl2 and Hif-1 [125]. There is some evidence that endogenous cannabinoids, acting through the $\mathrm{CB}_{1}$ receptor and $\mathrm{G}$ proteins, may protect neurons against glutamatemediated injury [126], as well as other injury mechanisms [127], suggesting $C_{1}$ receptors may be a potential therapeutic target for preconditioning. Neurons are protected from OGD after short-term exposure to hypoxia, and this protection lasts for up to $48 \mathrm{~h}$ after the preconditioning stimulus [128]. Pathways implicated as protective included the inhibition of caspase-12 after preconditioning, but not prolonged ischemia, and activation of multiple unfolded protein response pathways [128]. Finally, recent studies suggest that $14-3-3 \gamma$, a multifunctional scaffolding protein expressed in astrocytes in response to IPC, is also upregulated in neuronal cultures in response to OGD [103]. These findings suggest some conserved responses to hypoxia in several CNS cell types.

Loss of neuron-microglia contact appears to induce microglial activation through several mechanisms [19]. Neurons constitutively express $\mathrm{CX}_{3} \mathrm{CL} 1$ (fractalkine) on their surface, which suppresses microglial activation through the microglial receptor $\mathrm{CX}_{3} \mathrm{CR} 1$ [19]. After neuronal injury, including injury caused by ischemia, the loss of the fractalkine ligand expression on the surface of neurons results in enhanced microglial activation in several models of inflammation $[19,129]$. In the early stages following ischemia, however, deficiency in $\mathrm{CX}_{3} \mathrm{CR} 1$ signaling suppresses activation of microglia/macrophages, reduces neurotoxicity, and leads to a reduction in poststroke infarct volume $[130,131]$. The microglial/macrophage response to $\mathrm{CX}_{3} \mathrm{CL} 1 / \mathrm{CX}_{3} \mathrm{CR} 1$ signaling in ischemia likely evolves over time and the net effect of $\mathrm{CX}_{3} \mathrm{CL} 1 / \mathrm{CX}_{3} \mathrm{CR} 1$ signaling in IPC remains to be determined. In addition to this mechanism of communication and activation, OGD-stressed neurons in culture release glutamate, which, in turn, activates microglia in a group II metabotropic glutamate receptor-dependent manner [70]. These activated microglia, in turn, release TNF- $\alpha$, which induces neuron apoptosis in a caspase-3 dependent pathway [70]. There is a growing body of evidence that many neuronal subpopulations express TLRs, including TLR4 [132-135]. Mice with a defect in TLR4 are generally more resistant to CNS trauma [62], suggesting that TLR4 activation is detrimental to neuronal survival. Furthermore, the activation of microglia via TLR4 increases neuronal death in co-cultures of microglia and neurons [62], which may result in a possible "double hit" to neurons through activation of cell intrinsic programs in addition to neurotoxic microglial responses. The connections between astrocytes and neurons, astrocytes and microglia, and neurons and microglia create multiple regulatory levels that allow for microglia to regulate the CNS environment and respond to a variety of potential problems ranging from infection to cell death and mechanical trauma.

\section{Progenitor Cells}

Adult neurogenesis occurs in well-characterized neurogenic niches, such as the subventricular zone of the lateral ventricle and the subgranular zone of the dentate gyrus in the hippocampus, in both the normal adult brain and the ischemia-injured brain [136]. After acute ischemia, proliferation of these, and other, progenitor cells in the CNS is enhanced [88, 136, 137], and, similarly, shorter pulses of ischemia to induce preconditioning effects also result in the proliferation of progenitors [137, 138], with an increase of up to 4-fold observed after preconditioning in one study [137]. Interestingly, when proliferation was attenuated by administration of 
methylazoxymethanol acetate or ganciclovir, the preconditioning effect of a short ischemic event (15 min MCAO) was abolished [137]. These data suggest that the proliferation of progenitors is an effector of IPC-mediated neuroprotection.

Although some of these proliferative cells in the hippocampus may differentiate into $\mathrm{NeuN}^{+}$cells [138], the identity of other proliferating cells in different regions of the brain has not been fully determined, but there is evidence that microvascular pericytes proliferate in response to ischemia [139], as do reactive astrocytes [140]. We have observed in a model of IPC (15 min MCAO) that the number of microglia substantially and significantly increases in the ipsilateral hemisphere of a preconditioned mouse (Fig. 1). These ipsilateral microglia express cell proliferation markers and their genomic profile skews heavily towards cellular proliferation and DNA replication (J.R. Weinstein and A. McDonough, unpublished observations), Some of these proliferating cells may be local microglial progenitors or the self-renewal of a local microglial population. More work is necessary to characterize the molecular identity of these proliferating microglia and determine if they are, in fact, microglial progenitors induced by IPC. Future studies will also need to determine if these proliferating microglia are critical mediators of IPC-induced neuroprotection against subsequent prolonged ischemia/stroke. Once these are understood, therapies can be designed and tested to engage and modulate endogenous proliferative responses to ischemia.

\section{Peripheral Immune Cells}

The spleen is a peripheral immune system organ that responds to ischemic injury by releasing multiple immune cell types, resulting in a profound shrinkage of the spleen after stroke [141, 142]. The timing of the release of immune cells is swift, usually within 1 to 3 days [143]. However, the splenic response to stroke is generally regarded as destructive, with splenectomy providing marked protection against ischemic injury and resulting in a decreased infarct volume [144-147], in part by reducing neuroinflammation [144], which appears to be mediated primarily by IFN- $\gamma$ [147]. The spleen contains $\mathrm{T}$ cells, B cells, natural killer cells, and monocytes/macrophages [148], and splenectomy reduces the infiltration of all these cell types [144], leaving it unclear which cell types are responsible for the neurodegenerative effects of peripheral immune cells observed after stroke.

T cells play multiple roles in the pathophysiology of stroke, ranging from detrimental to protective, and are present in both acute and delayed phases of ischemia [19, 53, 149]. Unprimed $\mathrm{T}$ cells contribute to tissue damage in an antigen-independent manner, through unknown signaling mechanisms that may involve IFN- $\gamma$ and/or release of reactive oxygen species [19]. $\gamma \delta \mathrm{T}$ cells respond swiftly to ischemia and are regarded as detrimental, largely through their production of cytotoxic cytokines, including IL-17 [19, 150]. There is also a possibility that $\mathrm{CD} 4^{+}$and $\mathrm{CD} 8^{+}$helper $\mathrm{T}$ cells may become sensitized against CNS antigens, such as myelin basic protein, which may worsen stroke outcome, a phenomenon reviewed more extensively by Iadecola and Anrather [19]. Additionally, $\mathrm{CD}^{+}$helper $\mathrm{T}$ cells produce neurotoxic cytokines such as IFN- $\gamma$ and IL-4 [53]. Although the overall response of T cells is generally considered to exert neurotoxic effects [53], some subpopulations of $\mathrm{T}$ cells can function protectively in the context of ischemia. These T cells are activated through TGF- $\beta$ signaling from astrocytes [21] and/or macrophages [74]. TGF- $\beta$ promotes the development of regulatory T cells producing IL-10, which is protective in experimental stroke [19]. IL-10 also inhibits T helper 1 cell and Thelper 2 cell responses and shifts the cellular immune response towards neuroprotection [19].

The role of the spleen and peripheral immune cells, including $\mathrm{T}$ cells, has been largely uncharacterized in the context of preconditioning. In our studies of IPC, we have observed infiltration of innate immune cells that include Ly6 $\mathrm{C}^{\text {hi }}$ monocytes/macrophages, which are inflammatory cells that migrate to injured tissues, and Ly6 $\mathrm{C}^{\mathrm{lo}}$ monocytes/macrophages, which patrol resting and normal vasculature and have primarily antiinflammatory functions (Fig. 1). Both of these cell types are present in a reservoir of cells within the red pulp of the spleen and can be mobilized in response to inflammation [151].

Some evidence suggests that peripheral macrophages play a key role in stroke pathophysiology $[58,76]$; however, their role in IPC is unknown. Macrophages infiltrate the infarct border zone within hours of stroke onset and they undergo differentiation from a proinflammatory to a noninflammatory profile, which facilitates tissue repair [74, 152]. Macrophages are the primary producers of osteopontin, which may have neuroprotective and repair-promoting effects in CNS injury, including ischemia [58]. Recently published evidence suggests that osteopontin is critical for the polarization of astrocytes and establishment of an astrocytic barrier (glial scar) in the outer part of the ischemic core [58], suggesting another layer of interaction between immune cells and astrocytes. Furthermore, this study implicated osteopontin stimulation of astrocytes in the reestablishment of the BBB after ischemia [58].

Neutrophils accumulate swiftly after permanent MCAO [153], suggesting these are early responders to ischemia, and may be activated by IPC as well. Our own ex vivo flow cytometry findings indicate an increase in the number of neutrophils in the ipsilateral cortex after 15 min MCAO (IPC) (Fig. 1). Little is known about the contribution, if any, of neurophils to IPC-mediated neuroprotection. In stroke models, neutrophils begin to accumulate rapidly after MCAO, prior to neuronal death, and are thought to be responsible for the progression from tissue ischemia to cerebral infarction through various mechanisms, including secretion of MMPs and BBB disruption, obstruction of microcirculation in capillaries, and release of inflammatory cytokines [153]. 


\section{Summary}

The phenomenon of preconditioning can be achieved through a multitude of molecular effectors, which are present in a number of different cell types, including endogenous CNS cells such as microglia, astrocytes, and neurons, as well as infiltrating immune cells, such as macrophages and $\mathrm{T}$ cells. Astrocytes provide largely metabolic support to neurons under conditions of ischemia (Fig. 2), and function as an important link in the cross-talk between the local immune system (microglia) and other CNS cells (neurons) (Fig. 2). Preconditioning primes each cell type for a prolonged ischemic event in distinct and complementary ways; astrocytes are primed to provide increased metabolic support to neurons, neuronal metabolism shifts to adapt to conditions of low oxygen, and microglia also provide support to neurons and astrocytes to regulate the above processes. Microglia are also capable of directly interacting with, and influencing, neurons via a variety of signaling pathways, including responses to molecules directly released by neurons themselves. Interestingly, there are many conserved molecular mediators and pathways activated in all these cell types in response to preconditioning stimuli, although it is clear that some signaling pathways are more potent in specific cell types (i.e., TLR4 signaling in microglia) than in others. The combined outcome of preconditioning depends on the stimulus, for example preconditioning with LPS induces a primarily immune response, while preconditioning through ischemia induces shifts in metabolic activities and also activates the immune system. These cell type-specific differential responses represent a potential opportunity for therapeutic targeting in acute stroke, as well as in prevention of stroke injury in patients at risk of stroke. Further characterization of the cellular and molecular mechanisms underlying IPC may lead to novel therapeutic approaches in both the carefully selected "at immediate risk for stroke" patient populations studied in several recent large clinical trials and also more broadly for ameliorating brain injury in the general acute ischemic stroke patient population.

Acknowledgments We thank Dr. Shahani Noor and Richard Lee for assistance with ex vivo flow cytometry, as well as Thu Le and Jamie Colman for mouse middle cerebral artery surgeries.

Required Author Forms Disclosure forms provided by the authors are available with the online version of this article.

\section{References}

1. Gidday, J.M., Cerebral preconditioning and ischaemic tolerance. Nat Rev Neurosci, 2006. 7(6): p. 437-448.

2. Kariko, K., D. Weissman, and F.A. Welsh, Inhibition of toll-like receptor and cytokine signaling - a unifying theme in ischemic tolerance. J Cereb Blood Flow Metab, 2004. 24(11): p. 12881304.
3. Zhang, J., Z.J. Yang, J.A. Klaus, et al., Delayed tolerance with repetitive transient focal ischemic preconditioning in the mouse. Stroke, 2008. 39(3): p. 967-974.

4. Dirnagl, U., K. Becker, and A. Meisel, Preconditioning and tolerance against cerebral ischaemia: from experimental strategies to clinical use. Lancet Neurol, 2009. 8(4): p. 398-412.

5. Marsh, B., S.L. Stevens, A.E. Packard, et al., Systemic lipopolysaccharide protects the brain from ischemic injury by reprogramming the response of the brain to stroke: a critical role for IRF3. J Neurosci, 2009. 29(31): p. 9839-9849.

6. Stenzel-Poore, M.P., S.L. Stevens, J.S. King, et al., Preconditioning reprograms the response to ischemic injury and primes the emergence of unique endogenous neuroprotective phenotypes: a speculative synthesis. Stroke, 2007. 38(2 Suppl.): p. 680-685.

7. Moncayo, J., G.R. de Freitas, J. Bogousslavsky, et al., Do transient ischemic attacks have a neuroprotective effect? Neurology, 2000. 54(11): p. 2089-2094.

8. Wegener, S., B. Gottschalk, V. Jovanovic, et al., Transient ischemic attacks before ischemic stroke: preconditioning the human brain? A multicenter magnetic resonance imaging study. Stroke, 2004. 35(3): p. 616-621.

9. Stevens, S.L., K.B. Vartanian, and M.P. Stenzel-Poore, Reprogramming the response to stroke by preconditioning. Stroke, 2014. 45(8): p. 2527-2531.

10. Garcia-Bonilla, L., C. Benakis, J. Moore, et al., Immune mechanisms in cerebral ischemic tolerance. Front Neurosci, 2014. 8: p. 44.

11. Hamner, M.A., Z. Ye, R.V. Lee, et al., Ischemic preconditioning in white matter: magnitude and mechanism. J Neurosci, 2015. 35(47): p. 15599-15611.

12. Pradillo, J.M., D. Fernandez-Lopez, I. Garcia-Yebenes, et al., Tolllike receptor 4 is involved in neuroprotection afforded by ischemic preconditioning. J Neurochem, 2009. 109(1): p. 287-294.

13. Stevens, S.L., P.Y. Leung, K.B. Vartanian, et al., Multiple preconditioning paradigms converge on interferon regulatory factordependent signaling to promote tolerance to ischemic brain injury. J Neurosci, 2011. 31(23): p. 8456-8463.

14. Stenzel-Poore, M.P., S.L. Stevens, and R.P. Simon, Genomics of preconditioning. Stroke, 2004. 35(11 Suppl. 1): p. 2683-2686.

15. Simon, R.P., R. Meller, A. Zhou, et al., Can genes modify stroke outcome and by what mechanisms? Stroke, 2012. 43(1): p. 286291.

16. Murphy, K., P. Travers, M. Walport, et al., Janeway's immunobiology. 2012, New York: Garland Science.

17. Weinstein, J.R., I.P. Koerner, and T. Moller, Microglia in ischemic brain injury. Future Neurol, 2010. 5(2):p. 227-246.

18. Garden, G.A. and T. Moller, Microglia biology in health and disease. J Neuroimmune Pharmacol, 2006. 1(2): p. 127-37.

19. Iadecola, C. and J. Anrather, The immunology of stroke: from mechanisms to translation. Nat Med, 2011. 17(7): p. 796-808.

20. Norden, D.M., P.J. Trojanowski, E. Villanueva, et al., Sequential activation of microglia and astrocyte cytokine expression precedes increased iba-1 or GFAP immunoreactivity following systemic immune challenge. Glia, 2016. 64(2): p. 300-16.

21. Trendelenburg, G. and U. Dirnagl, Neuroprotective role of astrocytes in cerebral ischemia: focus on ischemic preconditioning. Glia, 2005. 50(4): p. 307-320.

22. Liu, M. and N.J. Alkayed, Hypoxic preconditioning and tolerance via hypoxia inducible factor (HIF) 1alpha-linked induction of P450 2C11 epoxygenase in astrocytes. J Cereb Blood Flow Metab, 2005. 25(8): p. 939-948.

23. Cramer, T.,Y. Yamanishi, B.E. Clausen., et al., HIF-1alpha is essential for myeloid cell-mediated inflammation. Cell, 2003. 112(5): p. 645-657. 
24. Makela, S.M., P. Osterlund, and I. Julkunen, TLR ligands induce synergistic interferon-beta and interferon-lambdal gene expression in human monocyte-derived dendritic cells. Mol Immunol, 2011. 48(4): p. 505-515.

25. Malmgaard, L., Induction and regulation of IFNs during viral infections. J Interferon Cytokine Res, 2004. 24(8): p. 439-454.

26. Stenzel-Poore, M.P., S.L. Stevens, Z. Xiong., et al., Effect of ischaemic preconditioning on genomic response to cerebral ischaemia: similarity to neuroprotective strategies in hibernation and hypoxiatolerant states. Lancet, 2003. 362(9389): p. 1028-1037.

27. Norden, D.M., P.J. Trojanowski, E. Villanueva, et al., TGFbeta produced by IL-10 redirected astrocytes attenuates microglial activation. Glia, 2014. 62(6): p. 881-895.

28. Gliem, M., M. Schwaninger, and S. Jander, Protective features of peripheral monocytes/macrophages in stroke. Biochim Biophys Acta, 2016. 1862(3): p. 329-338.

29. Marsh, B.J., S.L. Stevens, B. Hunter, et al., Inflammation and the emerging role of the toll-like receptor system in acute brain ischemia. Stroke, 2009. 40(3 Suppl.): p. S34-S37.

30. Dave, K.R., I. Saul, R. Prado, et al., Remote organ ischemic preconditioning protect brain from ischemic damage following asphyxial cardiac arrest. Neurosci Lett, 2006. 404(1-2): p. 170175 .

31. Ren, C., X. Gao, G.K. Steinberg, et al., Limb remotepreconditioning protects against focal ischemia in rats and contradicts the dogma of therapeutic time windows for preconditioning. Neuroscience, 2008. 151(4): p. 1099-1103.

32. Hausenloy, D.J., L. Candilio, and D.M. Yellon, Remote ischemic preconditioning and cardiac surgery. N Engl J Med, 2016. 374(5): p. 491-492.

33. Meybohm, P., D. Hasenclever, and K. Zacharowski, Remote ischemic preconditioning and cardiac surgery. N Engl J Med, 2016. 374(5): p. 491.

34. Healy DA, E. Boyle, D. McCartan, et al., A multicenter pilot randomized controlled trial of remote ischemic preconditioning in major vascular surgery. Vasc Endovasc Surg 2015;49(8):220 227

35. Gonzalez, N.R., M. Connolly, J.R. Dusick, et al., Phase I clinical trial for the feasibility and safety of remote ischemic conditioning for aneurysmal subarachnoid hemorrhage. Neurosurgery, 2014. 75(5): p. 590-598.

36. Laiwalla, A.N., Y.C. Ooi, R. Liou, et al., Matched cohort analysis of the effects of limb remote ischemic conditioning in patients with aneurysmal subarachnoid hemorrhage. Transl Stroke Res, 2016. 7(1): p. 42-48.

37. Meller R, Simon RP. A critical review of mechanisms regulating remote preconditioning-induced brain protection. J Appl Physiol (1985) 2015;119(10):1135-1142.

38. Barone, F.C., R.F. White, P.A. Spera, et al., Ischemic preconditioning and brain tolerance: temporal histological and functional outcomes, protein synthesis requirement, and interleukin-1 receptor antagonist and early gene expression. Stroke, 1998. 29(9): p. 1937-50; discussion 1950-1.

39. Kalakech, H., S. Tamareille, S. Pons, et al., Role of hypoxia inducible factor-1alpha in remote limb ischemic preconditioning. J Mol Cell Cardiol, 2013. 65: p. 98-104.

40. Hibert, P., D. Prunier-Mirebeau, O. Beseme, et al., Apolipoprotein a-I is a potential mediator of remote ischemic preconditioning. PLoS One, 2013. 8(10): p. e77211.

41. Hibert, P., D. Prunier-Mirebeau, O. Beseme, et al., Modifications in rat plasma proteome after remote ischemic preconditioning (RIPC) stimulus: identification by a SELDI-TOF-MS approach. PLoS One, 2014. 9(1): p. e85669.

42. Konstantinov, I.E., S. Arab, R.K. Kharbanda, et al., The remote ischemic preconditioning stimulus modifies inflammatory gene expression in humans. Physiol Genomics, 2004. 19(1): p. 143150.

43. Wei, D., C. Ren, X. Chen, et al., The chronic protective effects of limb remote preconditioning and the underlying mechanisms involved in inflammatory factors in rat stroke. PLoS One, 2012. 7(2): p. e30892.

44. Kirino, T., T. Nakagomi, H. Kanemitsu, et al., Ischemic tolerance. Adv Neurol, 1996. 71: p. 505-11.

45. Gesuete R, A.E. Packard, K.B. Vartanian, et al. Poly-ICLC preconditioning protects the blood-brain barrier against ischemic injury in vitro through type I interferon signaling. J Neurochem 2012;123(Suppl. 2):75-85.

46. Leung, P.Y., S.L. Stevens, A.E. Packard, et al., Toll-like receptor 7 preconditioning induces robust neuroprotection against stroke by a novel type I interferon-mediated mechanism. Stroke 2012. 43(5): p. 1383-1389.

47. Packard, A.E., P.Y. Leung, K.B. Vartanian, et al., TLR9 bone marrow chimeric mice define a role for cerebral TNF in neuroprotection induced by $\mathrm{CpG}$ preconditioning. J Cereb Blood Flow Metab, 2012. 32(12): p. 2193-2200.

48. Su, W., S. Hopkins, N.K. Nesser, et al., The p53 transcription factor modulates microglia behavior through microRNAdependent regulation of c-Maf. J Immunol, 2014. 192(1): p. 358-66.

49. Stark, G.R., How cells respond to interferons revisited: from early history to current complexity. Cytokine Growth Factor Rev, 2007. 18(5-6): p. 419-423.

50. Stark, G.R., I.M. Kerr, B.R. Williams, et al., How cells respond to interferons Annu Rev Biochem, 1998. 67: p. 227-264.

51. Khorooshi, R. and T. Owens, Injury-induced type I IFN signaling regulates inflammatory responses in the central nervous system. J Immunol, 2010. 185(2): p. 1258-1264.

52. Inacio, A.R., Y. Liu, B.H. Clausen, et al., Endogenous IFN-beta signaling exerts anti-inflammatory actions in experimentally induced focal cerebral ischemia. J Neuroinflammation, 2015. 12: p. 211.

53. Shichita, T., T. Ago, M. Kamouchi, et al., Novel therapeutic strategies targeting innate immune responses and early inflammation after stroke. J Neurochem, 2012. 123(Suppl. 2): p. 29-38.

54. Khorooshi, R., M.T. Morch, T.H. Holm, et al., Induction of endogenous Type I interferon within the central nervous system plays a protective role in experimental autoimmune encephalomyelitis. Acta Neuropathol, 2015. 130(1): p. 107-18.

55. Blackburn, D., S. Sargsyan, P.N. Monk, et al., Astrocyte function and role in motor neuron disease: a future therapeutic target? Glia, 2009. 57(12): p. 1251-64.

56. Jha, M.K., W.H. Lee, and K. Suk, Functional polarization of neuroglia: implications in neuroinflammation and neurological disorders. Biochem Pharmacol, 2016;103:116..

57. Rama Rao, K.V. and T. Kielian, Neuron-astrocyte interactions in neurodegenerative diseases: role of neuroinflammation. Clin Exp Neuroimmunol, 2015. 6(3): p. 245-263.

58. Gliem, M., K. Krammes, L. Liaw, et al., Macrophage-derived osteopontin induces reactive astrocyte polarization and promotes re-establishment of the blood brain barrier after ischemic stroke. Glia, 2015. 63(12): p. 2198-207.

59. Ejlerskov, P., J.G. Hultberg, J. Wang, et al., Lack of Neuronal IFNbeta-IFNAR Causes Lewy Body- and Parkinson's Disease-like Dementia. Cell, 2015. 163(2): p. 324-339.

60. Marsh BJ, Stenzel-Poore MP. Toll-like receptors: novel pharmacological targets for the treatment of neurological diseases. Curr Opin Pharmacol 2008;8(1):8-13.

61. Shichita, T., M. Ito, and A. Yoshimura, Post-ischemic inflammation regulates neural damage and protection. Front Cell Neurosci, 2014. 8: p. 319. 
62. Lehnardt, S., E. Schott, T. Trimbuch, et al., A vicious cycle involving release of heat shock protein 60 from injured cells and activation of toll-like receptor 4 mediates neurodegeneration in the CNS. J Neurosci, 2008. 28(10): p. 2320-31.

63. Zhan, X., C. Kim, and F.R. Sharp, Very brief focal ischemia simulating transient ischemic attacks (TIAs) can injure brain and induce Hsp70 protein. Brain Res, 2008. 1234: p. 183-197.

64. Venna, V.R., J. Li, S.E. Benashski, et al., Preconditioning induces sustained neuroprotection by downregulation of adenosine 5'monophosphate-activated protein kinase. Neuroscience, 2012. 201: p. 280-7.

65. Bahjat, F.R., R.L. Williams-Karnesky, S.G. Kohama, et al., Proof of concept: pharmacological preconditioning with a Toll-like receptor agonist protects against cerebrovascular injury in a primate model of stroke. J Cereb Blood Flow Metab, 2011. 31(5): p. 12291242.

66. Caso, J.R., J.M. Pradillo, O. Hurtado, et al., Toll-like receptor 4 is involved in brain damage and inflammation after experimental stroke. Circulation, 2007. 115(12): p. 1599-608.

67. Cao, C.X., Q.W. Yang, F.L. Lv, et al., Reduced cerebral ischemiareperfusion injury in Toll-like receptor 4 deficient mice. Biochem Biophys Res Commun, 2007. 353(2): p. 509-514.

68. Hayakawa, K., J. Qiu, and E.H. Lo, Biphasic actions of HMGB1 signaling in inflammation and recovery after stroke. Ann N Y Acad Sci, 2010. 1207: p. 50-57.

69. Yu, S., T. Zhao, M. Guo, et al., Hypoxic preconditioning upregulates glucose transport activity and glucose transporter (GLUT1 and GLUT3) gene expression after acute anoxic exposure in the cultured rat hippocampal neurons and astrocytes. Brain Res, 2008. 1211: p. 22-29.

70. Kaushal, V. and L.C. Schlichter, Mechanisms of microgliamediated neurotoxicity in a new model of the stroke penumbra. J Neurosci, 2008. 28(9): p. 2221-2230.

71. Amantea, D., G. Micieli, C. Tassorelli, et al., Rational modulation of the innate immune system for neuroprotection in ischemic stroke. Front Neurosci, 2015. 9: p. 147.

72. Rosenzweig, H.L., M. Minami, N.S. Lessov, et al., Endotoxin preconditioning protects against the cytotoxic effects of TNFalpha after stroke: a novel role for TNFalpha in LPSischemic tolerance. J Cereb Blood Flow Metab, 2007. 27(10): p. 1663-74.

73. Stevens, S.L., T.M. Ciesielski, B.J. Marsh, et al., Toll-like receptor 9: a new target of ischemic preconditioning in the brain. J Cereb Blood Flow Metab, 2008. 28(5): p. 1040-1047.

74. Gliem, M., A.K. Mausberg, J.I. Lee, et al., Macrophages prevent hemorrhagic infarct transformation in murine stroke models. Ann Neurol, 2012. 71(6): p. 743-52.

75. Macrez, R., C. Ali, O. Toutirais, et al., Stroke and the immune system: from pathophysiology to new therapeutic strategies. Lancet Neurol, 2011. 10(5): p. 471-480.

76. Dirnagl, U., Pathobiology of injury after stroke: the neurovascular unit and beyond. Ann N Y Acad Sci, 2012. 1268: p. 21-25.

77. Maki, T., K. Hayakawa, L.D. Pham, et al., Biphasic mechanisms of neurovascular unit injury and protection in CNS diseases. CNS Neurol Disord Drug Targets, 2013. 12(3): p. 302-315.

78. Benarroch, E.E., Microglia: Multiple roles in surveillance, circuit shaping, and response to injury. Neurology, 2013. 81(12): p. 10791088.

79. Michell-Robinson, M.A., et al., Roles of microglia in brain development, tissue maintenance and repair. Brain, 2015. 138(Pt 5): p. 1138-1159.

80. Ginhoux, F., M. Greter, M. Leboeuf, et al., Fate mapping analysis reveals that adult microglia derive from primitive macrophages. Science, 2010. 330(6005): p. 841-5.

81. Sedgwick, J.D., S. Schwender, H. Imrich, et al., Isolation and direct characterization of resident microglial cells from the normal and inflamed central nervous system. Proc Natl Acad Sci U S A, 1991. 88(16): p. 7438-7442.

82. Butovsky, O., M.P. Jedrychowski, C.S. Moore, et al., Identification of a unique TGF-beta-dependent molecular and functional signature in microglia. Nat Neurosci, 2014. 17(1): p. 131-43.

83. Olson, J.K. and S.D. Miller, Microglia initiate central nervous system innate and adaptive immune responses through multiple TLRs. J Immunol, 2004. 173(6): p. 3916-3924.

84. Lalancette-Hebert, M., G. Gowing, A. Simard, et al., Selective ablation of proliferating microglial cells exacerbates ischemic injury in the brain. J Neurosci, 2007. 27(10): p. 2596-605.

85. Nedergaard, M. and U. Dirnagl, Role of glial cells in cerebral ischemia. Glia, 2005. 50(4): p. 281-286.

86. van Rossum, D. and U.K. Hanisch, Microglia. Metab Brain Dis, 2004. 19(3-4): p. 393-411.

87. Cunningham, L.A., M. Wetzel, and G.A. Rosenberg, Multiple roles for MMPs and TIMPs in cerebral ischemia. Glia, 2005. 50(4): p. 329-339.

88. Umekawa, T., A.M. Osman, W. Han, et al., Resident microglia, rather than blood-derived macrophages, contribute to the earlier and more pronounced inflammatory reaction in the immature compared with the adult hippocampus after hypoxia-ischemia. Glia, 2015. 63(12): p. 2220-30.

89. Prinz, M., H. Schmidt, A. Mildner, et al., Distinct and nonredundant in vivo functions of IFNAR on myeloid cells limit autoimmunity in the central nervous system. Immunity, 2008. 28(5): p. 675-86.

90. Owens, T., R. Khorooshi, A. Wlodarczyk, et al., Interferons in the central nervous system: a few instruments play many tunes. Glia, 2014. 62(3): p. 339-55.

91. Costello, D.A. and M.A. Lynch, Toll-like receptor 3 activation modulates hippocampal network excitability, via glial production of interferon-beta. Hippocampus, 2013. 23(8): p. 696-707.

92. Tarassishin, L., H.S. Suh, and S.C. Lee, Interferon regulatory factor 3 plays an anti-inflammatory role in microglia by activating the PI3K/Akt pathway. J Neuroinflammation, 2011. 8: p. 187.

93. Brendecke, S.M. and M. Prinz, How type I interferons shape myeloid cell function in CNS autoimmunity. J Leukoc Biol, 2012. 92(3): p. 479-488.

94. Teige, I., Y. Liu, and S. Issazadeh-Navikas, IFN-beta inhibits T cell activation capacity of central nervous system APCs. J Immunol, 2006. 177(6): p. 3542-3553.

95. Kim, M.O., Q. Si, J.N. Zhou, et al., Interferon-beta activates multiple signaling cascades in primary human microglia. J Neurochem, 2002. 81(6): p. 1361-1371.

96. Jin, S., J. Kawanokuchi, T. Mizuno, et al., Interferon-beta is neuroprotective against the toxicity induced by activated microglia. Brain Res, 2007. 1179: p. 140-146.

97. Kawanokuchi, J., T. Mizuno, H. Kato, et al., Effects of interferonbeta on microglial functions as inflammatory and antigen presenting cells in the central nervous system. Neuropharmacology, 2004. 46(5): p. 734-742.

98. Tanaka, T., K. Murakami, Y. Bando, et al., Interferon regulatory factor 7 participates in the M1-like microglial polarization switch. Glia, 2015. 63(4): p. 595-610.

99. Satoh, T., O. Takeuchi, A. Vandenbon, et al., The Jmjd3-Irf4 axis regulates M2 macrophage polarization and host responses against helminth infection. Nat Immunol, 2010. 11(10): p. 936-944.

100. Salem, M., J.T. Mony, M. Lobner, et al., Interferon regulatory factor-7 modulates experimental autoimmune encephalomyelitis in mice. J Neuroinflammation, 2011. 8: p. 181.

101. Chan, A., R. Seguin, T. Magnus, et al., Phagocytosis of apoptotic inflammatory cells by microglia and its therapeutic implications: termination of CNS autoimmune inflammation and modulation by interferon-beta. Glia, 2003. 43(3): p. 231-242. 
102. Pang, Y., C.R. Chai, K. Gao, et al., Ischemia preconditioning protects astrocytes from ischemic injury through 14-3-3gamma. J Neurosci Res, 2015. 93(10): p. 1507-1518.

103. Li, L., A. Lundkvist, D. Andersson, et al., Protective role of reactive astrocytes in brain ischemia. J Cereb Blood Flow Metab, 2008. 28(3): p. 468-81.

104. Hossain, M.Z., J. Peeling, G.R. Sutherland, et al., Ischemiainduced cellular redistribution of the astrocytic gap junctional protein connexin43 in rat brain. Brain Res, 1994. 652(2): p. 311-22.

105. Nakase, T., T., S. Fushiki, G. Sohl, et al., Neuroprotective role of astrocytic gap junctions in ischemic stroke. Cell Commun Adhes, 2003. 10(4-6): p. 413-417.

106. Chen, Y. and R.A. Swanson, Astrocytes and brain injury. J Cereb Blood Flow Metab, 2003. 23(2): p. 137-149.

107. Freitas-Andrade, M. and C.C. Naus, Astrocytes in neuroprotection and neurodegeneration: the role of connexin 43 and pannexin1. Neuroscience, 2016. 323: p. 207-21.

108. Gorina, R., M. Font-Nieves, L. Marquez-Kisinousky, et al., Astrocyte TLR4 activation induces a proinflammatory environment through the interplay between MyD88-dependent NFkappaB signaling, MAPK, and Jak1/Stat1 pathways. Glia, 2011. 59(2): p. 242-255.

109. Hung, C.C., C.H. Lin, H. Chang, et al.,Astrocytic GAP43 Induced by the TLR4/NF-kappaB/STAT3 Axis Attenuates AstrogliosisMediated Microglial Activation and Neurotoxicity. J Neurosci, 2016. 36(6): p. 2027-2043.

110. Pan, L.N., W. Zhu, Y. Li, et al., Astrocytic Toll-like receptor 3 is associated with ischemic preconditioning-induced protection against brain ischemia in rodents. PLoS One, 2014. 9(6): p. e99526.

111. Lian, H., A. Litvinchuk, A.C. Chiang, et al., Astrocyte-microglia cross talk through complement activation modulates amyloid pathology in mouse models of Alzheimer's disease. J Neurosci, 2016. 36(2): p. 577-589.

112. Weinstein, J.R., Y. Quan, J.F. Hanson, et al., IgM-dependent phagocytosis in microglia is mediated by complement receptor 3, not Fcalpha/mu receptor. J Immunol, 2015. 195(11): p. 5309-5317.

113. Hong, S., V.F. Beja-Glasser, B.M. Nfonoyim, et al., Complement and microglia mediate early synapse loss in Alzheimer mouse models. Science, 2016.

114. Benarroch, E.E., Neuron-astrocyte interactions: partnership for normal function and disease in the central nervous system. Mayo Clin Proc, 2005. 80(10): p. 1326-1338.

115. Nakase, T. and C.C. Naus, Gap junctions and neurological disorders of the central nervous system. Biochim Biophys Acta, 2004. 1662(1-2): p. 149-158.

116. Wallraff, A., R. Kohling, U. Heinemann, et al., The impact of astrocytic gap junctional coupling on potassium buffering in the hippocampus. J Neurosci, 2006. 26(20): p. 5438-5447.

117. Guitart, K., G. Loers, F. Buck, et al., Improvement of neuronal cell survival by astrocyte-derived exosomes under hypoxic and ischemic conditions depends on prion protein. Glia, 2016. 64(6): p. 896-910.

118. Dodson, R.F., L.W. Chu, K.M. Welch, et al., Acute tissue response to cerebral ischemia in the gerbil. An ultrastructural study. J Neurol Sci, 1977. 33(1-2): p. 161-70.

119. del Zoppo, G.J., Inflammation and the neurovascular unit in the setting of focal cerebral ischemia. Neuroscience, 2009. 158(3): p. 972-982.

120. Rosenberg, G.A., E.Y. Estrada, and J.E. Dencoff, Matrix metalloproteinases and TIMPs are associated with blood-brain barrier opening after reperfusion in rat brain. Stroke, 1998. 29(10): p. 2189-2195.

121. Filosa, J.A., H.W. Morrison, J.A. Iddings, et al., Beyond neurovascular coupling, role of astrocytes in the regulation of vascular tone. Neuroscience, 2016. 323: p. 96-109.

122. Hayakawa, K., T. Nakano, K. Irie, et al., Inhibition of reactive astrocytes with fluorocitrate retards neurovascular remodeling and recovery after focal cerebral ischemia in mice. $\mathrm{J}$ Cereb Blood Flow Metab, 2010. 30(4): p. 871-82.

123. Nawashiro, H., M. Brenner, S. Fukui, et al., High susceptibility to cerebral ischemia in GFAP-null mice. J Cereb Blood Flow Metab, 2000. 20(7): p. 1040-1044.

124. Wang, X., K. Kang, S. Wang, et al. Focal cerebral ischemic tolerance and change in blood-brain barrier permeability after repetitive pure oxygen exposure preconditioning in a rodent model. $\mathrm{J}$ Neurosurg 2016;1-10.

125. Jin, K., X.O. Mao, M.W. Eshoo, et al., cDNA microarray analysis of changes in gene expression induced by neuronal hypoxia in vitro. Neurochem Res, 2002. 27(10): p. 1105-1112.

126. Shen, M., T.M. Piser, V.S. Seybold, et al., Cannabinoid receptor agonists inhibit glutamatergic synaptic transmission in rat hippocampal cultures. J Neurosci, 1996. 16(14): p. 4322-34.

127. Nagayama, T., A.D. Sinor, R.P. Simon, et al., Cannabinoids and neuroprotection in global and focal cerebral ischemia and in neuronal cultures. J Neurosci, 1999. 19(8): p. 2987-2995.

128. Bickler, P.E., J.P. Clark, P. Gabatto, et al., Hypoxic preconditioning and cell death from oxygen/glucose deprivation co-opt a subset of the unfolded protein response in hippocampal neurons. Neuroscience, 2015. 310: p. 306-321.

129. Cardona, A.E., E.P. Pioro, M.E. Sasse, et al., Control of microglial neurotoxicity by the fractalkine receptor. Nat Neurosci, 2006. 9(7): p. $917-924$

130. Tang, Z., Y. Gan, Q. Liu, et al., CX3CR1 deficiency suppresses activation and neurotoxicity of microglia/macrophage in experimental ischemic stroke. J Neuroinflammation, 2014. 11: p. 26.

131. Fumagalli, S., C. Perego, F. Ortolano, et al., CX3CR1 deficiency induces an early protective inflammatory environment in ischemic mice. Glia, 2013. 61(6): p. 827-42.

132. Helley, M.P., W. Abate, S.K. Jackson, et al., The expression of Toll-like receptor 4, 7 and co-receptors in neurochemical subpopulations of rat trigeminal ganglion sensory neurons. Neuroscience, 2015. 310: p. 686-98.

133. Lafon, M., F. Megret, M. Lafage, et al., The innate immune facet of brain: human neurons express TLR-3 and sense viral dsRNA. J Mol Neurosci, 2006. 29(3): p. 185-94.

134. Mishra, B.B., P.K. Mishra, and J.M. Teale, Expression and distribution of Toll-like receptors in the brain during murine neurocysticercosis. J Neuroimmunol, 2006. 181(1-2): p. 46-56.

135. Wadachi, R. and K.M. Hargreaves, Trigeminal nociceptors express TLR-4 and CD14: a mechanism for pain due to infection. J Dent Res, 2006. 85(1): p. 49-53.

136. Marlier, Q., S. Verteneuil, R. Vandenbosch, et al., Mechanisms and functional significance of stroke-induced neurogenesis. Front Neurosci, 2015. 9: p. 458.

137. Maysami, S., J.Q. Lan, M. Minami, et al., Proliferating progenitor cells: a required cellular element for induction of ischemic tolerance in the brain. J Cereb Blood Flow Metab, 2008. 28(6): p. 1104-1113.

138. Naylor, M., K.K. Bowen, K.A. Sailor, et al., Preconditioninginduced ischemic tolerance stimulates growth factor expression and neurogenesis in adult rat hippocampus. Neurochem Int, 2005. 47(8): p. 565-572.

139. Nakagomi, T., S. Kubo, A. Nakano-Doi, et al., Brain vascular pericytes following ischemia have multipotential stem cell activity to differentiate into neural and vascular lineage cells. Stem Cells, 2015. 33(6): p. 1962-1974.

140. Lang, B., H.L. Liu, R. Liu, et al., Astrocytes in injured adult rat spinal cord may acquire the potential of neural stem cells. Neuroscience, 2004. 128(4): p. 775-783.

141. Offner, H., S. Subramanian, S.M. Parker, et al., Splenic atrophy in experimental stroke is accompanied by increased regulatory $\mathrm{T}$ cells and circulating macrophages. J Immunol, 2006. 176(11): p. 6523-6531. 
142. Vendrame, M., C. Gemma, K.R. Pennypacker, et al., Cord blood rescues stroke-induced changes in splenocyte phenotype and function. Exp Neurol, 2006. 199(1): p. 191-200.

143. Gelderblom, M., F. Leypoldt, K. Steinbach, et al., Temporal and spatial dynamics of cerebral immune cell accumulation in stroke. Stroke, 2009. 40(5): p. 1849-1857.

144. Ajmo, C.T., Jr., D.O. Vernon, L. Collier, et al., The spleen contributes to stroke-induced neurodegeneration. J Neurosci Res, 2008. 86(10): p. 2227-2234.

145. Das, M., C.C. Leonardo, S. Rangooni, et al., Lateral fluid percussion injury of the brain induces CCL20 inflammatory chemokine expression in rats. J Neuroinflammation, 2011. 8: p. 148.

146. Jin, R., X. Zhu, L. Liu, et al., Simvastatin attenuates strokeinduced splenic atrophy and lung susceptibility to spontaneous bacterial infection in mice. Stroke, 2013. 44(4): p. 1135-43.

147. Seifert, H.A., C.C. Leonardo, A.A. Hall, et al., The spleen contributes to stroke induced neurodegeneration through interferon gamma signaling. Metab Brain Dis, 2012. 27(2): p. 131-141.
148. Seifert, H.A. and K.R. Pennypacker, Molecular and cellular immune responses to ischemic brain injury. Transl Stroke Res, 2014. 5(5): p. 543-553.

149. Becker, K.J., Activation of immune responses to brain antigens after stroke. J Neurochem, 2012. 123(Suppl. 2): p. 148-155.

150. Shichita, T., Y. Sugiyama, H. Ooboshi, et al., Pivotal role of cerebral interleukin-17-producing gammadeltaT cells in the delayed phase of ischemic brain injury. Nat Med, 2009. 15(8): p. 946-950.

151. Swirski, F.K., M. Nahrendorf, M. Etzrodt, et al., Identification of splenic reservoir monocytes and their deployment to inflammatory sites. Science, 2009. 325(5940): p. 612-6.

152. Gliem, M., D. Hermsen, N. van Rooijen, et al., Secondary intracerebral hemorrhage due to early initiation of oral anticoagulation after ischemic stroke: an experimental study in mice. Stroke, 2012. 43(12): p. 3352-3357.

153. Huang, J., U.M. Upadhyay, and R.J. Tamargo, Inflammation in stroke and focal cerebral ischemia. Surg Neurol, 2006. 66(3): p. 232-245. 